\title{
New method for estimating the refractive index of optical materials in spectrally selective elements
}

\author{
A.A. Manko', G.S. Svechnikov ${ }^{2}$ \\ ${ }^{1}$ Odessa National Communication Academy, \\ 1, Kovalska str., 65029 Odessa, Ukraine \\ ${ }^{2} V$. Lashkaryov Institute of Semiconductor Physics, NAS of Ukraine, \\ 41, prospect Nauky, 03028 Kyiv, Ukraine
}

\begin{abstract}
A numerical simulation of the scattering indicatrix in optical spectral-selective cylindrical form elements has been performed. As it follows from the results of the calculations, the shape of the scattering indicatrix of these elements is unambiguously determined by the refractive index of material from which they are made. Location of angular peaks in the indicatrix in combination with its shape allows to estimate the refractive index with sufficiently high degree of accuracy.
\end{abstract}

Keywords: optical cylindrical element, light scattering, Monte Carlo method, indicatrix of scattering, refractive index.

Manuscript received 23.12.15; revised version received 31.03.16; accepted for publication 08.06.16; published online 06.07.16.

\section{Introduction}

In Ref. [1], the dispersion characteristics of the scattering function inherent to the optical spectralselective elements of the cylindrical shape with the refractive index that did not exceed 2.25 were considered. At the same time, a number of optical materials with refractive indexes that exceed this value exist. For example, the refractive index of $\mathrm{ZnTe}$ in the infrared range is 5.3. In addition, progress in the development of nanotechnology provides creation (synthesis) of the so-called heterogeneous environment. The heterogeneous materials consist of a matrix semiconductor or dielectric with metal, dielectric, semiconductor, ferromagnetic and ferroelectric nanoparticles, size of which is much smaller than the wavelength of incident electromagnetic radiation. When using different materials for nanoparticles, it is possible to change the refractive index of the heterogeneous environment within wide limits and in a predetermined wavelength range.

Thus, studying the features of optical characteristics of the spectrally selective elements with the refractive index value higher than that of typical optical materials [2] is essential. In particular, it is interesting to study the indicatrix of scattering of optical elements of the cylindrical shape to apply them to demultiplexing in optical signal systems [3], as well as to ascertain a relationship between the refractive index of these elements and their indicatrix of scattering.

\section{Solution of the problem and numerical results}

In this paper, the same cylindrical optical elements were considered and the same principles of modeling as in [1] were applied. As it was discussed, the input light flux 
consisted of parallel beams of incoherent light. The reflection coefficient $C_{R}$ at the interface between optical media is described by the expression obtained in [4]:

$$
C_{R}=\frac{n_{1}^{2} \cos 2 \theta-2 n_{1}^{2} \cos \theta \sqrt{\left(n_{2} / n_{1}\right)^{2}-\sin ^{2} \theta}+n_{2}^{2}}{n_{1}^{2} \cos 2 \theta+2 n_{1}^{2} \cos \theta \sqrt{\left(n_{2} / n_{1}\right)^{2}-\sin ^{2} \theta}+n_{2}^{2}},
$$

where $n_{1}, n_{2}$ are the refractive indexes of air and glass, respectively, $\theta$ is the angle of incidence.

To simulate the light scattering, the principles set out in [5] were used. Using the proposed methods, it was calculated the indicatrix of scattering of the optical elements with different refractive indices. In particular, the scattering function calculations were carried out for the refractive index close to 1.46 (fused silica, $\mathrm{SiO}_{2}$ ).

Fig. 1 shows the scattering indicatrix of the optical cylindrical element with the refractive index $n_{2}=1.458$. Direction of the input beam coincides with that of the $x$ axis. As can be seen from the figure, the indicatrix has four intensity peaks arranged symmetrically with respect to the $x$-axis at the angles $\varphi_{1}= \pm 100^{\circ}$ and $\varphi_{2}= \pm 155^{\circ}$. The normalized values of intensity of these peaks are 0.62 , and 1.0 , respectively.

With a decrease in the refractive index $n_{2}$, the angle $\varphi_{1}$ increases, and the angle $\varphi_{2}$ is reduced, accordingly. Thus, these parameters are characterized by dispersive properties regarding the refractive index $n$. These properties are reflected by the values of the derivative component of the first peak $-3.49 \mathrm{rad}$, and that of the second one $1.745 \mathrm{rad}$, which is half as high as the first one. Numerical values represent shift of the peak (in radians) when changing the refractive index by one.

With a decrease in the refractive index down to the value 1.33 (the refractive index of water), the first and second peaks move and occupy the angular positions $129^{\circ}$ and $139^{\circ}$, respectively (Fig. 2), which coincides within the calculating program resolution ability with the data obtained by other authors [6] by using other methods of calculation. These are the angles at which in the drops of rain the first and second rainbows (can be observed) [6]. This fact confirms correctness of the proposed method.

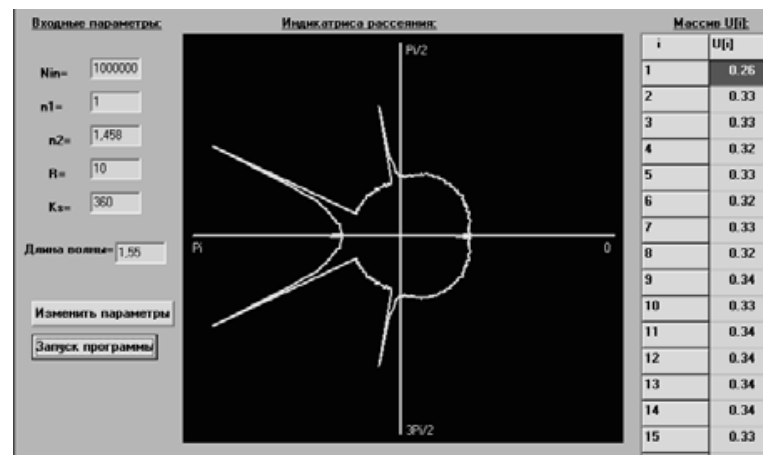

Fig. 1. The scattering indicatrix of a cylindrical optical element with the refractive index $n_{2}=1.458$.
With an increase in the refractive index to the value 2.4 , which lies in the vicinity of the diamond refractive index $\left(n_{2}=2.42\right)$, the scattering indicatrix is transformed as illustrated in Fig. 3.

It can be seen that there are four peaks which are arranged symmetrically with respect to the $x$-axis. The angular positions of the large peaks have the values close to $\pm 12^{\circ}$. The increase in the refractive index up to $n_{2}=2.5$ insignificantly transforms the shape of the indicatrix and changes the angular position of the large peaks down to $\pm 9^{\circ}$ (Fig. 4).

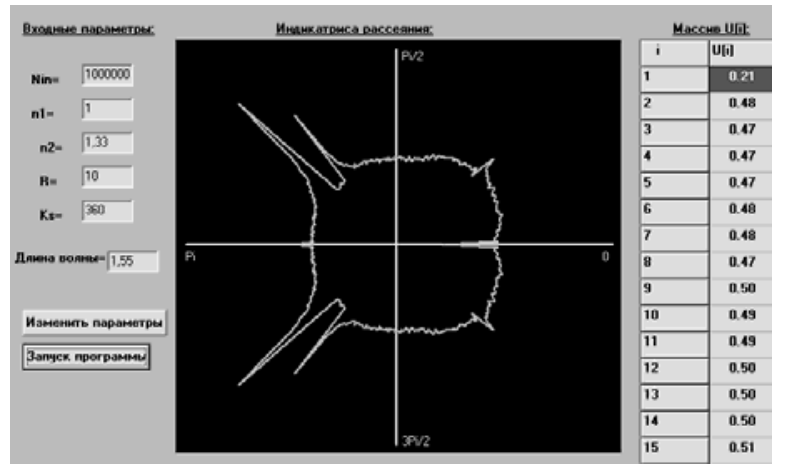

Fig. 2. The scattering indicatrix of a cylindrical optical element with the refractive index $n_{2}=1.33$.

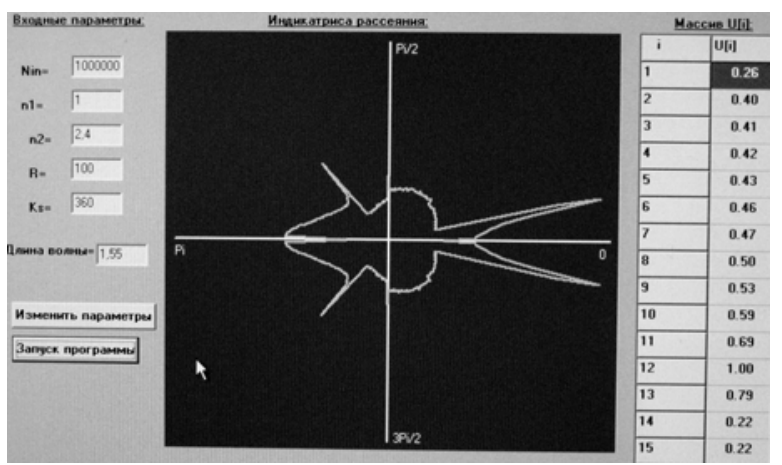

Fig. 3. The scattering indicatrix of a cylindrical optical element with the refractive index $n_{2}=2.4$.

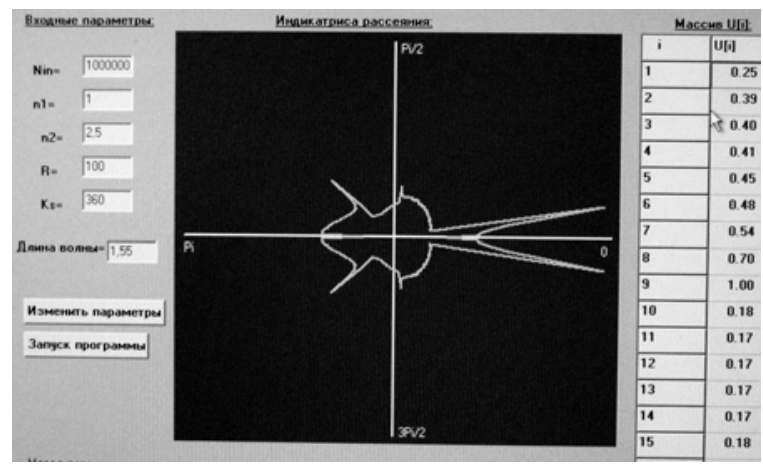

Fig. 4. The scattering indicatrix of a cylindrical optical element with the refractive index $n_{2}=2.5$. 
Further increase in the refractive index up to $n_{2}=2.6$ changes the angular position of the large peaks down to the values $\pm 6^{\circ}$ (Fig. 5).

Thus, the value of the derivative $\partial \varphi / \partial n$ in this range of values inherent to the refractive index is $0.52 \mathrm{rad}$. In its turn, the refractive index of the optical element depends on the wavelength of light $\lambda$ in accordance with the Selmeyer formula:

$n(\lambda)=\sqrt{1+\sum_{i=1}^{3} \frac{A_{i} \cdot \lambda^{2}}{\lambda^{2}-\left(L_{i}\right)^{2}}}$,

where $A_{i}$ and $L_{i}$ are Selmeyer's factors. When using the optical element as the spectrally selective one for separation of optical channels, quality of demultiplexing is determined by angular dispersion properties of the element in relation to the wavelength $\partial \varphi / \partial \lambda$. In turn, this derivative, as a derivative of a composite function, can be presented as $\frac{\partial \varphi}{\partial n} \frac{\partial n}{\partial \lambda}$. The value $\partial \varphi / \partial n$ is defined by the design features of the optical element, in our case, by cylindrical shape, and the value $\partial n / \partial \lambda$ is defined by glass properties of the element:

$\frac{d n}{d \lambda}=-\left(\frac{\lambda}{n}\right)\left[\sum_{i=1}^{3} \frac{A_{i} \cdot L_{i}^{2}}{\left(\lambda^{2}-L_{i}^{2}\right)^{2}}\right]$.

Thus, to perform operations of demultiplexing, it is necessary to choose material of a spectrally selective element not only by the value of refractive index but also by the magnitude of its derivative $\partial n / \partial \lambda$ in the operation wavelength range. Solution of the problem to improve the dispersion properties can be explained differently - by choosing the operation wavelength range in which the derivative $\partial n / \partial \lambda$ takes absolute extreme values. For this purpose, it is necessary to investigate the behavior of the second derivative $\partial^{2} n / \partial \lambda^{2}$ :

$\frac{d^{2} n}{d \lambda^{2}}=\frac{1}{n}\left[\sum_{i=1}^{3} \frac{A_{i} l_{i}^{2}\left(3 \lambda^{2}+L_{i}^{2}\right)}{\left(\lambda^{2}-L_{i}^{2}\right)^{3}}-\left(\frac{d n}{d \lambda}\right)^{2}\right]$,

to determine the wavelength at which it becomes zero.

As shown by the results of research, formulating tasks for optimization and solving the problems with this optimization of dispersion characteristics inherent to spectrally selective optical elements makes sense, if the scattering indicatrix has quite sharp and significant peaks with noticeable angular dispersion.

To determine the upper limit of the operation range for the refractive index, calculations of the scattering function were performed at higher values of the refractive index. Thus, with an increase in the values of the refractive index up to 2.8, both peaks (see Fig. 5) are combined into one (Fig. 6).
If there is a subsequent increase in $n_{2}$, the change in the nature of transformation of the scattering indicatrix begins. In this case, the refractive index increase is not accompanied by angular displacement of the peaks within the range from 0 to $\pi \mathrm{rad}$ as it took place at the lower values of $n_{2}$.

With an increase in the refractive index to the value of $n_{2}=3.8$, redistribution of the peak intensity in the opposite direction (Fig. 7) takes place. This makes it impossible to use optical elements as spectrally selective devices in a given range of values of the refractive indices.

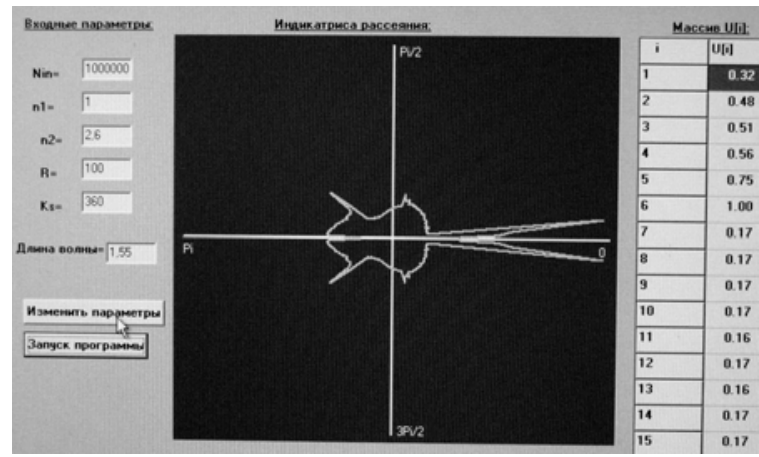

Fig. 5. The scattering indicatrix of a cylindrical optical element with the refractive index $n_{2}=2.6$.

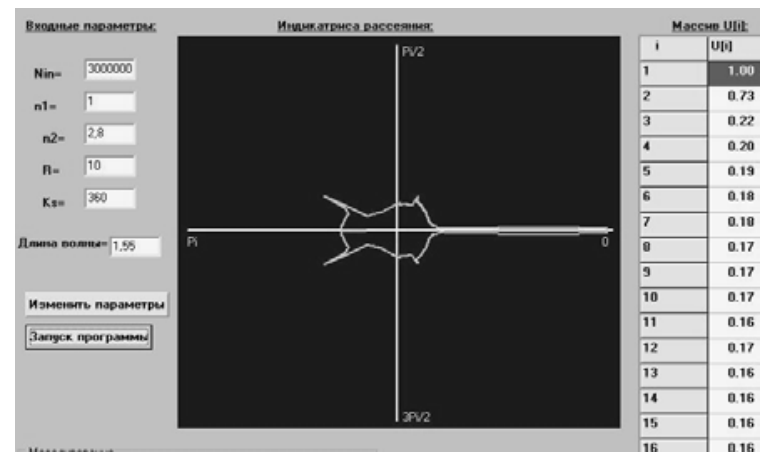

Fig. 6. The scattering indicatrix of a cylindrical optical element with the refractive index $n_{2}=2.8$.

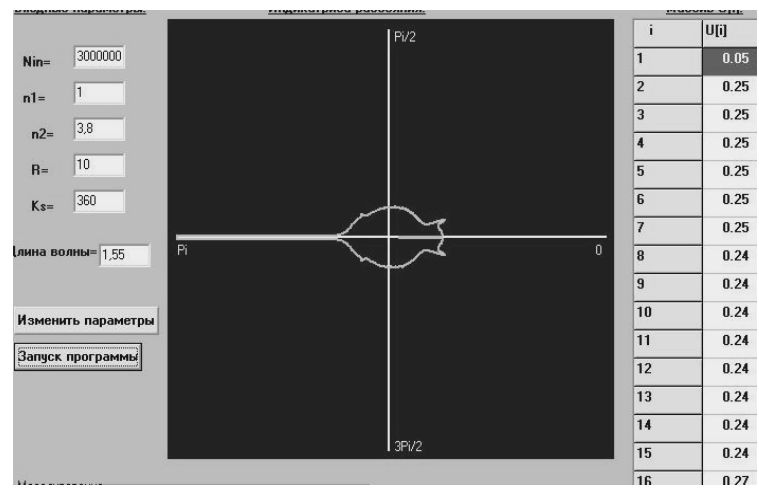

Fig. 7. The scattering indicatrix of a cylindrical optical element with the refractive index $n_{2}=3.8$. 


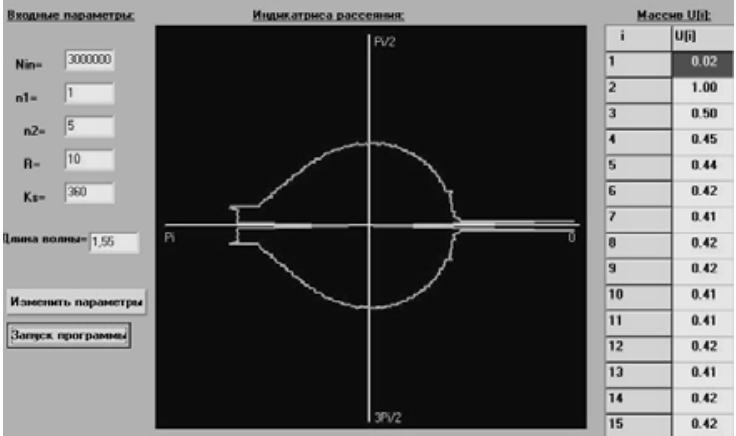

Fig. 8. The scattering indicatrix of a cylindrical optical element with the refractive index $n_{2}=5.0$.

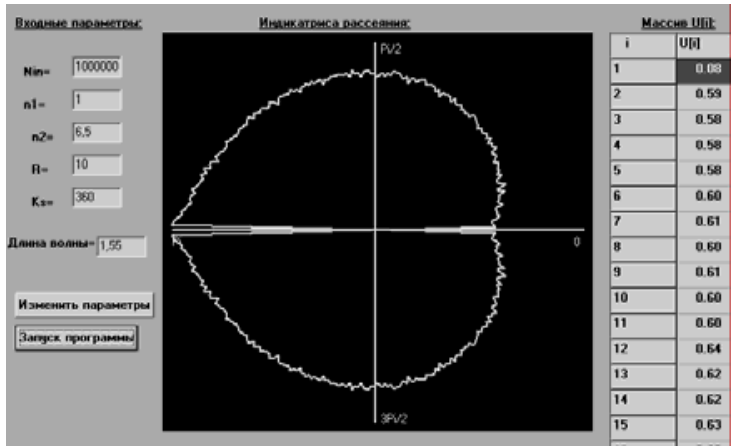

Fig. 9. The scattering indicatrix of a cylindrical optical element with the refractive index $n_{2}=6.5$.

A further increase in the refractive index up to 5.0 leads to converse redistribution of energy peaks (Fig. 8). However, the relative magnitude of the peaks is less than of the average level of scattering intensity.

The increase in the refractive index up to the values of 5.6...6.5 leads to redistribution of the intensity of scattered light, thus these peaks disappear, also the indicatrix has the form shown in Fig. $9\left(n_{2}=6.5\right)$.

The relative intensity of the scattered signal changes insignificantly within the angular limits. Only in the narrow range of $\pm 1^{\circ}$ in the direction of the incidence of the light beam and $\pm 1^{\circ}$ in the opposite direction, it drops down to 0.07 and 0.02 , respectively. Thus, a cylindrical optical element at the large values of the refractive index $\left(n_{2} \geq 5.6\right)$ with a good degree of uniformity and an absence of peaks operates in a quasiuniform function of the light scattering mode.

A further increase in the refractive index confirms this result. Practical usage of elements with a quasiuniform indicatrix seems appropriate for local systems of atmospheric optical communication with a number of facilities disposed in different directions. These elements may be used for both transmitting and receiving devices. An analogue using these elements is the use of an omnidirectional radio antenna.

\section{Conclusion}

As shown by the results of the research, the use of a cylindrical optical element for the spectral angular selection is limited by the refractive index values approximately up to $n_{2}=2.8$. In this range of the refractive index values, the shape of the scattering indicatrix and the angular positions of peaks unambiguously define the value of the refractive index of optical element material. The accuracy of determination of the refractive index primarily depends on the structural arrangement of the device for measuring the intensity distribution of light scattered by the optical element.

With an increase in the value of the refractive index up to 5.6 and above, the optical element begins to function in the angular light scattering mode with a fairly good degree of uniformity. Thus, in this mode, the elements can be used in local multipoint systems for laser atmospheric communication in transmitting and receiving devices. When using the optical elements as the spectral-selective angular devices, it is necessary to choose specific glass with high dispersion properties. For that, an analysis of the first and second derivatives of the refractive index with respect to the wavelength is obligatory.

\section{References}

1. V.A. Manko, A.A. Manko, Optical spectralselective elements using effect of the light scattering // Proc. $10^{\text {th }}$ Intern. Conf. "Laser and Fiber-Optical Networks Modeling (LFNM'2010)", September 12-14, 2010, Sevastopol, Crimea, Ukraine, p. 170-171.

2. I.Ya. Bubis, W.A. Waydenbach, I.I. Duchopel et al., Handbook of Technologist-Optics / Ed. S.M. Kuznetsov. Mechanical Eng., Leningrad, 1983 (in Russian).

3. N.N. Slepov, Optical multiplexers and demultiplexers WDM systems // Electronics: Science, Technology, Business, №8, p. 42-47 (2004), in Russian.

4. M. Born, E. Wolf, Principles of Optics. Pergamon Press, Oxford, London, Edinburgh, New York, 1965.

5. G.A. Sukach, V.A. Manko, A.A. Manko, Method of calculation of multilayer optical filters using thin films" / Proc. $8^{\text {th }}$ Int. Conf. on Laser and Fiber-Optical Networks Modelling (LFNM 2006), Kharkiv, Ukraine, June 29 July 01, 2006, p. 452-454.

6. H.C. van de Hulst, Light Scattering by Small Particles. New York, John Wiley \& Sons, Inc. London, Chapman \& Hall, Ltd. 1957. 